\title{
Editoriale
}

\section{Per aspera ad astra: 2012, un anno molto importante per Financial Reporting}

\author{
Alberto Quagli
}

Per Financial Reporting il 2012, terzo anno di vita della rivista, sarà molto importante per il grosso sforzo che faremo sulla strada della internazionalizzazione, sotto vari profili.

Anzitutto, come Comitato Scientifico abbiamo deciso infatti che dal 2012 le nuove sottomissioni alla rivista dovranno essere solo in inglese per ampliare la platea potenziale tanto di lettori quanto di autori. Già adesso la rivista ha in corso di referaggio oltre 20 paper in inglese, inviati tanto da autori italiani che stranieri. I primi numeri del 2012 conterranno ancora articoli in italiano i cui referaggi sono attualmente in corso, ma l'obiettivo è di avere una rivista tutta in inglese per la fine dell'anno, per poter poi puntare in futuro ad un accreditamento presso le migliori Journal List internazionali.

In secondo luogo, stiamo agendo sulla composizione del Comitato Scientifico, inserendo qualificati colleghi stranieri molto attivi nella ricerca. Gli ultimi entrati sono Joachim Gassen (Università di Berlino) ed Araceli Mora (Università di Valencia) e miriamo ad inserirne alcuni altri nel corso dell'anno.

Infine, dal 2012 abbiamo deciso di svolgere il convegno annuale della rivista esclusivamente in inglese. Questo anno Financial Reporting terrà il suo convegno a Napoli, Università Parthenope, il 14 e 15 giugno, sotto la supervisione organizzativa del collega Raffaele Fiume e sarà il primo completamente in inglese (informazioni e inscrizioni tramite sito web http://www.frworkshop.com).

Nello stesso senso stiamo cercando di aprire alla internazionalizzazione anche i contatti con i principali regulators europei per ospitare nella rivista il dibattito in merito alle questioni principali sullo standard setting europeo.

Obiettivi ambiziosi, che in presenza di risorse (sempre) scarse, sono oltremodo sfidanti. Ma siamo fortemente motivati.

Il 2012 sarà importante anche sotto il profilo della valutazione della ricerca da parte delle istituzioni ministeriali italiane (ANVUR) e della associazioni scientifiche (AIDEA). Come molti di voi già sanno, nel 2012 inizierà la valutazione quadriennale della ricerca $(\mathrm{VQR})$ da parte dell'ANVUR che grandi conseguenze avrà 
sugli Atenei, determinando una quota rilevante dei finanziamenti concessi dal MIUR. Nello stesso senso, l'ANVUR costruirà i ranking dei docenti universitari basati proprio sui risultati della ricerca che saranno l'elemento essenziale per i nuovi concorsi di accesso e di progresso nella carriera universitaria. A tale scopo, è compito delle associazioni scientifiche portare all'attenzione dell'ANVUR le proprie liste di merito, ossia gli elenchi delle riviste e monografie ordinate secondo la qualità, così come sono valutati dall'intera comunità di studiosi di un certo settore disciplinare. Per quanto riguarda noi aziendalisti, l'AIDEA (Accademia Italiana di Economia Aziendale) sta compiendo grossi sforzi in tre direzioni. Anzitutto ha sviluppato un journal rating delle riviste internazionali di ambito aziendale già nel 2007 e nel 2012 procederà ad approvare la nuova versione, sempre basata sulla suddivisione in quattro fasce delle rivista, prendendo spunto dal JQL internazionale (http://www.harzing.com/jql.htm). In secondo luogo, AIDEA ha predisposto una lista di riviste italiane accreditate che nel prossimo futuro sarà basata su una individuazione delle diverse fasce di merito. In terzo luogo, sta predisponendo dei requisiti per l'accreditamento di monografie e collane, sempre basandosi sulla logica della peer review e della formazione di adeguati comitati scientifici.

Pur apprezzando il grande sforzo di AIDEA, ritengo necessario svolgere una specifica riflessione riguardante l'approccio di derivare il Journal Rating AIDEA dal Journal Quality List. Quest'ultimo archivio trae spunto da varie liste provenienti o da associazioni scientifiche o da singole/gruppi di università e business school. In prospettiva, ritengo che debba essere AIDEA stessa a proporre una propria lista, sviluppata con un rigoroso sondaggio presso i propri associati, che possa ambire ad entrare nel JQL, lista che tenga conto anche delle riviste italiane che più si impegnano sul fronte della qualità e della internazionalizzazione per promuoverle anche all'estero. Dobbiamo far crescere il panorama nazionale, senza schiacciarci oltre il tempo strettamente necessario su prodotti altrui. Il processo verso l'internazionalizzazione deve basarsi su un reciproco scambio che consiste sia nel recepire quanto di meglio c'è oltre frontiera, sia nel far conoscere all'estero quanto di meglio c'è in Italia. Limitarsi indefinitamente solo al primo flusso sarebbe una politica quantomeno miope.

Dopo questo "accorato appello", è il caso di ricordare il successo del workshop Accounting Renaissance svoltosi all'Università di Venezia dal 3 al 5 novembre del 2011, del quale Financial Reporting era co-sponsor assieme alla International Association of Accounting Education and Research (IAAER). Una bella sintesi del convegno organizzato dai colleghi Stefano Zambon e da Luciano Olivotto è disponibile nella EAA newsletter (The official newsletter of the European Accounting Association) n. 4/2011. La qualità dei paper e dei guest speakers è stata veramente elevata, come raramente capita di vedere in Italia. Ed un bel po' di papers sono stati sottoposti a Financial Reporting.

A tutti, lettori, referees, autori e staff di Financial Reporting, i migliori auguri per il nuovo anno.

AlbertoQuagli

Editor 